\title{
Driver Detecting System based on Brain-Computer Interface
}

\author{
Hu Jian-feng Mu Zhen-dong Wang Ping \\ Institute of Information Technology, Jiangxi University of Technology, Nanchang 330098, China
}

Keyword: Brain-Computer Interface (BCl); Electroencephalogram (EEG); Driver; Drowsiness

\begin{abstract}
BCI technology has a lot of sophisticated technology, the use of brain-computer interface to calculate the state of the driver's driving a lot of testing is to avoid driving errors occur due to an accident, we use mobile brain-computer interface device driver by four imagine species to detect motion, simple design feature extraction and classification algorithm, the results show the recognition rate can be well imagined results identify driving.
\end{abstract}

\section{Introduction}

Drowsiness can affect a driver's abilities. When the drive long time or product driving fatigue, there may be drowsiness. When driver is drowsiness, driver find it hard to concentrate, once there is an emergency, the driver may be difficult to control and traffic accident will be happened. One important factors of traffic accident is drowsiness, so the detection of driver drowsiness is becoming an important research field in car safety. The traditional detection method is mainly the way of image detection and head movement, this paper attempts to judge by detection of driver's neural activities and develop brain-computer interface model.

Brain-computer interface $(\mathrm{BCI})$ is a device that enables the use of Electroencephalogram (EEG) to communicate with others or to control machines. As computerized systems are becoming one of the main tools for making people's lives easier and with the ongoing growth in the BCI field, it is becoming more important to understand brain waves and analyze EEG signals. EEG is the process of measuring the brain's neural activity as electrical voltage fluctuations along the scalp as a result of the current flows in brain's neurons. BCI captures EEG signals in conjunction with a specific user activity then uses different signal processing algorithms to translate these records into control commands for different machine and computer applications.

\section{Methods}

A. EEG Data Acquisition: In this study, EEG data from 8 channels were collected using the Nuamps EEG acquisition system, digitally sampled at $250 \mathrm{~Hz}$ with a resolution of 16 bits. EEG recordings from all channels were bandpass filtered from $1 \mathrm{~Hz}$ to $30 \mathrm{~Hz}$.

B. Preprocessing: A MATLAB script was written to analyze the filtered EEG signals and it was found that a subject imagines opening and closing a fist (or both fists/feet) and keeps doing this for 4.1 seconds then he takes a rest for the duration of 4.2 seconds. This means that each two-minute EEG run includes 15 events that are separated by a short neutral period where the subject relaxes. As the Physionet dataset was sampled at 160 samples per second, each vector includes 656 samples of the original recorded EEG signal. And because we used the available records for 100 subjects, our subset included 18000 vectors representing imagined left fist, right fist, both fists, and both feet movements. 
An additional 1500 vectors were extracted from the one-minute baseline run (with eyes open) and another 1500 vectors from the one-minute baseline run with eyes closed. So, the total number of extracted samples (events) was 12000 samples.

C. Feature Selection and Extraction: The original EEG is a signal domain, the features are hidden between the various noise data, through the original EEG feature extraction speed and accuracy will be affected, in order to improve the speed of analysis and analysis accuracy, can be converted to the original EEG power spectrum, the frequency analysis. A simple way to estimate the power spectrum of the stochastic process is a direct request sampling DFT, and then takes the results of the square of the amplitude. Such a method is called period gram

A length L of the signal $x_{L}[n]$ is estimated period gram PSD as follow

$$
P_{x x}(f)=\frac{\left|X_{L}(f)\right|^{2}}{f_{s} L}
$$

Where $X_{L}(f)$ is defined using the FFT matlab inside without normalization coefficients, so dividing L.

$$
X_{L}(f)=\sum_{n=0}^{L-1} x_{L}[n] e^{-2 \pi j f n / f s}
$$

The actual calculation of $X_{L}(f)$ may be performed using FFT, and only on a limited frequency. Applications in practice are most period gram PSD estimation to calculate the N-point:

$$
\begin{aligned}
& P_{x x}(f)=\frac{\left|X_{L}(f)\right|^{2}}{f_{s} L}, f_{k}=\frac{k f_{s}}{N}, k=1,2,3 \ldots, N-1 \\
& X_{L}\left(f_{k}\right)=\sum_{n=0}^{L-1} x_{L}[n] e^{-2 \pi j f h / N}
\end{aligned}
$$

the EEG signal used the method described as formula 4 to PSD, and then Fisher distance used to extract the EEG features, the method can be describe as follow:

Fisher distance $F_{i, j}$ is calculated as

$$
F_{i, j}=\frac{\left(\mu_{i}-\mu_{j}\right)^{2}}{\sigma_{i}^{2}+\sigma_{j}^{2}}
$$

Where $F_{i, j}$ is Fisher' distance between the subject I and the subject j. $\mu$ and $\sigma$ are the mean and the standard deviation of the feature they correspond to.

D. Classification: all subjects' feature, we select the outstanding sample components features set, and then use sample matching method to distinguish the classification of online collect samples.

The sample matching method described as follow:

Use the above method extract the feature and then calculate classification boundaries as follow: 


$$
T=\frac{\sum_{i=1}^{n} t_{i}}{n}
$$

Where $\mathrm{T}$ is the classification boundary, and $\mathrm{n}$ is the number of learning samples, $\mathrm{t}_{\mathrm{i}}$ is featuring of sample i.

$\mathrm{n}$ is EEG signal, and output is the kind of this EEG signal, in this paper the kind of EEG was describe as 1,2,3 and 4 where 1 is represent left fist,, 2 is represent right fist, 3 is represent both fists, and 4 is represent both feet movements. The output calculated as following:

$$
x=\left\{\begin{array}{lll}
1 & c l_{1}=\max \left\{c l_{k}\right\} & k=1,2,3,4 \\
2 & c l_{1}=\max \left\{c l_{k}\right\} & k=1,2,3,4 \\
3 & c l_{1}=\max \left\{c l_{k}\right\} & k=1,2,3,4 \\
4 & c l_{1}=\max \left\{c l_{k}\right\} & k=1,2,3,4
\end{array}\right.
$$

Where $\mathrm{cl}_{\mathrm{k}}$ is the absolute value of the feature minus classification boundary, and $\mathrm{cl}_{\mathrm{k}}$ calculated as following:

$$
\mathrm{clk}=|\mathrm{ti}-\mathrm{Ti}|
$$

The system of driver detecting designed as figure 1:

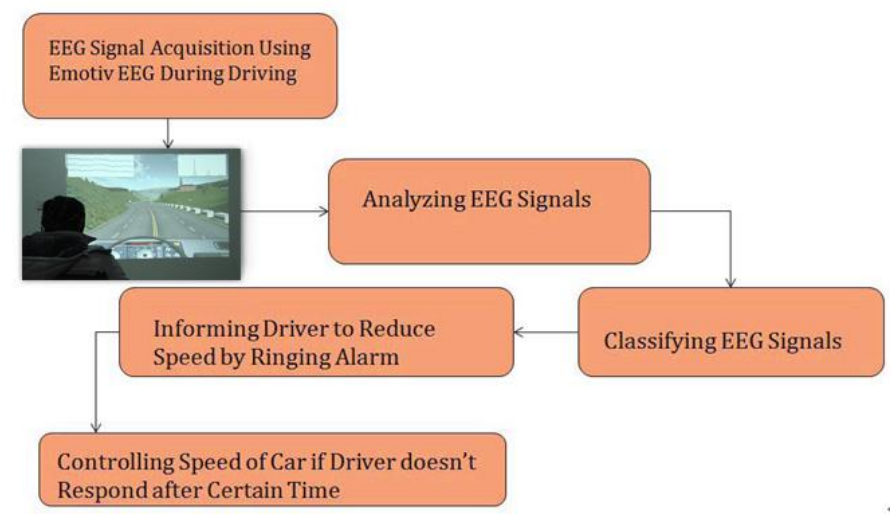

Figure. 1 system of driver detecting design

\section{Results}

In this paper, a total of 20,000 valid samples collected, which include 18000 sample of subjects to imagine left fist, right fist, both fists, and both feet movements, 2000 baseline reference samples. The sample was divided 18000 into 11000 learning samples and 7000 test samples, the use of the above described features extraction and classification algorithm to identify the sample rate, the recognition rate of the four imagine movement were: $82.33 \%, 81.05 \%, 73.45 \%$ and $85.87 \%$, showed that the use of EEG can recognize good driving condition.

\section{Acknowledgment}

This work was supported by Natural Science Foundation of Jiangxi Province [No 20142BAB207008] and project of Science and Technology Department of Jiangxi Province[No 2013BBE50051] 


\section{References}

[1] Donoghue JP. Connecting cortex to machines: recent advances in brain interfaces [J]. Nature Neuroscience, 2002: 1085 - 1088.

[2] Levine S, Huggins J, BeMent S, et al. Identification of electrocorticogram patterns as the basis for a direct brain interface [J]. Journal of Clinical Neurophysiology, 1999: 439-447.

[3] Wolpaw J, Birbaumer N, McFarland D, et al. Brain-computer interfaces for communication and control [J]. Clinical Neurophysiology, 2002: 767-791.

[4] Hu JF, Mu ZD. Smart Car Security System by Using Biometrics Based on EEG [J]. 2013 2nd International Conference on Frontiers of Energy and Environment Engineering, 2013: 1047-1049.

[5] Fuletra JD, Bosamiya D. A Survey on Driver's Drowsiness Detection Techniques [J]. International Journal on Recent and Innovation Trends in Computing and Communication, 2013, 816-819.

[6] Saini V, Saini R. Driver Drowsiness Detection System and Techniques: A Review [J]. International Journal of Computer Science and Information Technologies, 2014, 4245-4249.

[7] Lee BG, Chung WY. A Smartphone-Based Driver Safety Monitoring System Using Data Fusion [J]. Sensors, 2012, 17536-17552.

[8] Vasavi D, Krishnaiah RV. Online detection of drowsiness using brain and visual information [J]. International Journal of Instrumentation Electrical Electronics Engineering, 2013, 31-34. 